\title{
Tumor cell-derived Timp-1 is necessary for maintaining metastasis-promoting Met-signaling via inhibition of Adam-10
}

\author{
Florian Schelter • Martina Grandl • Bastian Seubert • \\ Susanne Schaten · Stephanie Hauser • Michael Gerg • \\ Carla Boccaccio $\cdot$ Paolo Comoglio $\cdot$ Achim Krüger
}

Received: 30 March 2011 / Accepted: 11 July 2011 / Published online: 26 July 2011

(C) Springer Science+Business Media B.V. 2011

\begin{abstract}
In many different tumor entities, increased expression of tissue inhibitor of metalloproteinases-1 (Timp-1) is associated with poor prognosis. We previously reported in mouse models that elevated systemic levels of Timp-1 induce a gene expression signature in the liver microenvironment increasing the susceptibility of this organ to tumor cells. This host effect was dependent on increased activity of the hepatocyte growth factor (Hgf)/ hepatocyte growth factor receptor (Met) signaling pathway. In a recent study we showed that Met signaling is regulated by Timp- 1 as it inhibits the Met sheddase A disintegrin and metalloproteinase-10 (Adam-10). The aim of the present study was to elucidate whether the metastatic potential of tumor cells benefits from autocrine Timp-1 as well and involves Adam-10 and Met signaling. In a syngeneic murine model of experimental liver metastasis Timp-1 expression and Met signaling were localized within metastatic colonies and expressed by tumor cells. Knock down of tumor cell Timp-1 suppressed Met signaling in metastases and inhibited metastasis formation and tumor cellscattering in the liver. In vitro, knock down of tumor cell Timp-1 prevented Hgf-induced Met phosphorylation. Consequently, knock down of Met sheddase Adam-10 triggered auto-phosphorylation and responsiveness to Hgf. Accordingly, Adam-10 knock down increased Met
\end{abstract}

F. Schelter · M. Grandl - B. Seubert - S. Schaten - S. Hauser M. Gerg · A. Krüger $(\bowtie)$

Institut für Experimentelle Onkologie und Therapieforschung des Klinikums rechts der Isar, Technische Universität München, Ismaninger Straße 22, 81675 Munich, Germany

e-mail: achim.krueger@1rz.tu-muenchen.de

C. Boccaccio - P. Comoglio

IRCC-Institute for Cancer Research at Candiolo, Università di

Torino, 10060 Candiolo, Italy phosphorylation in metastatic foci and induced tumor cell scattering into the surrounding liver parenchyma. In conclusion, these findings show that tumor cell-derived Timp1 acts as a positive regulator of the metastatic potential and support the concept that proteases and their natural inhibitors, as members of the protease web, are major players of signaling during normal homeostasis and disease.

Keywords Hgf - Liver metastasis - Met · Protease web · Timp-1

$\begin{array}{ll}\text { Abbreviations } \\ \text { DAPI } & \text { 4',6-Diamidine-2-phenylindole } \\ \text { Hgf } & \text { Hepatocyte growth factor } \\ \text { i.v. } & \text { Intraveneous } \\ \text { Met } & \text { Hepatocyte growth factor receptor } \\ \text { MMP } & \text { Matrix metalloproteinase } \\ \text { qRT-PCR } & \text { Quantitative real-time polymerase chain } \\ & \text { reaction } \\ \text { rec } & \text { Recombinant } \\ \text { SF } & \text { Scatter factor } \\ \text { sh } & \text { Small hairpin } \\ \text { TBS } & \text { 2-Amino-2-(hydroxymethyl)-propane-1,3- } \\ & \begin{array}{l}\text { diole-buffered saline } \\ \text { Timp }\end{array} \\ \text { Tissue inhibitor of metalloproteinases } \\ \text { X-Gal } & \text { 5-Bromo-4-chloro-3-indolyl- } \beta \text {-D- } \\ & \text { galactopyranoside }\end{array}$

\section{Introduction}

Many independent studies in a variety of tumor entities have shown that high levels of tissue inhibitor of 
metalloproteinases-1 (Timp-1) correlate with bad prognosis for cancer patients [1-4]. Timp-1 is an endogenous broad spectrum inhibitor of many metalloproteinases [5], including most matrix metalloproteinases (MMPs), which were also shown to correlate with the progression of tumors [6-9]. While it is widely accepted that elevated proteolytic activity should support invasive events of the metastatic cascade such as intra- and extravasation [6, 9], the fact that some MMPs, such as MMP-8 [10] and MMP-9 [11] were identified to play also a tumor-inhibiting role, depending on the specific microenvironmental context, excited some drawback to this concept.

Even more paradoxical seemed to be the finding that MMP inhibition by synthetic inhibitors [12] and the natural inhibitor Timp-1 [13, 14] promotes metastasis. This was one explanation of the failure of clinical trials with broad spectrum MMP inhibition [8, 15]. However, recent progress in the protease research field revealed that proteases and their endogenous inhibitors comprise a complex interacting network which, based on the check and balances of proteases and their inhibitors, regulates tissue homeostasis in health and disease $[15,16]$. Consequently, it is necessary to investigate each protease and protease inhibitor in a context in order to understand its possible functions [17]. In addition, this may explain supposedly paradoxical observations such as Timp-1 correlating with bad prognosis [1-4].

We recently started to elucidate the molecular basis of this paradoxon. We showed that elevated levels of Timp-1 in mice provoke severe changes in tissue homeostasis in the liver, which correlated with a dramatic change in the gene expression profile in this organ [14]. This Timp1-induced alteration of homeostasis in the liver increased the susceptibility of this organ to metastasis [14]. Notably, we observed a Timp-1-induced scattering pattern of single metastatic cells of the well-established lacZ-tagged L-CI.5s metastasis model $[18,19]$ throughout the liver parenchyma [14]. This increase in tumor cell scattering correlated with the Scatter factor (SF or hepatocyte growth factor (Hgf) [20])-activated hepatocyte growth factor receptor (Met) signaling pathway [14], which is generally regarded as the major inducer of tumor cell scattering [21-23]. Hgf/Met signaling pathway seems to be the central pathway mediating the pro-metastatic effects of Timp-1, as inhibition of Met signaling by a Met-specific synthetic tyrosine kinase inhibitor abrogates the pro-metastatic effects of Timp- 1 in vivo [14]. Elevated levels of Timp-1 lead to accumulation of the Met-receptor and increased signaling [14]. We and others recently found that a disintegrin and metalloproteinase-10 (Adam-10) [24], which is inhibited by Timp-1 [25], is a sheddase of the Met-receptor [26, 27]. Knock down and inhibition studies revealed that Adam-10 is involved in the regulation of Met-signaling [26].
Massive dissemination of tumor cells into and scattering within different vital organs often occurs in the late phase of tumor disease and finally leads to disturbed and disrupted functional integrity of the metastasized organ [28]. Therefore the metastatic potential of tumor cells is in most cases the major determinant of the lethality of the disease [29, 30]. The lacZ-tagged L-CI.5s liver metastasis model is very suitable to examine in detail such late phases of metastasis. It is one of the few models generating metastatic colonies in the liver, a target organ of virtually all malignant tumors, in spontaneous and experimental metastasis assays [14, 18, 19]. Specifically, in this model, single cell extravasation, growth of a colony, and secondary invasion of single tumor cells into the surrounding liver tissue can be monitored with high resolution and reproducibility of the experimental time frame [18, 19]. This scattered phenotype of invasive tumor cells migrating into the tissue surrounding the primary metastatic colony is dependent on activation of Met signaling in the tumor cells [19]. Therefore it is most suitable to investigate the relevance of a molecular pathway in vivo.

The goal of the present study was to test whether tumor cells, which induce Timp-1 expression in their microenvironment while establishing a metastatic colony, can autonomously use the above-described mechanism to exhibit their metastatic potential. To this end we prevented induction of Timp- 1 expression in tumor cells with shRNA technology. Indeed, the metastatic potential of the L-CI.5s cells was dependent on endogenous Timp-1 regulating Met-signaling and tumor cell scattering in vivo via Adam10. This is a new and unexpected function of tumor cellderived Timp-1 in the context of tumor progression and supports the notion that members of the protease web, such as Timp-1, regulate disease-relevant signaling.

\section{Materials and methods}

\section{Cells and viruses}

HEK293 human embryonic kidney cells as well as lacZtagged L-CI.5s T-lymphoma cells were cultured as described previously [31, 32]. Stable knock down of Timp-1 in L-CI.5s cells was achieved using lentiviral shRNAs. shRNA oligos targeting murine Timp-1 (Eurogentec) were designed with BamHI and EcoRI ends and annealed according to standard protocols (Timp-1 targeting sequence: 5'-AAGAGTTTCT CATCACGGGCC- $3^{\prime}$ ). shRNA oligos targeting murine Adam-10 were designed with BamHI and EcoRI ends and annealed according to standard protocols (Adam-10 targeting sequence: $5^{\prime}$-AATTCAAGCTTCCAAAAAACCTACG AATGAAGAGGGACTCT- $3^{\prime}$ ). Annealed small interfering RNAs were cloned into the retroviral vector $\mathrm{pSiren-RetroQ}$ 
(Clontech-Takara Bio Europe, Saint-Germain-en-Laye, France). Retroviral particles were produced as described elsewhere [18]. Transduction was done as described previously [18].

RNA isolation, reverse transcription, and quantitative real-time polymerase chain reaction (qRT-PCR)

RNA from cells was isolated using the TRI reagent (Sigma-Aldrich Chemie) and from tissues using the PureYield RNA Midiprep System (Promega, Mannheim, Germany) according to the manufacturers' protocols. Reverse transcription was performed with the High Capacity cDNA Reverse Transcription Kit (Applied Biosystems, Frankfurt am Main, Germany) according to the provided protocol. qRT-PCR was done as described previously [19] using inventoried primers and probes (Applied Biosystems).

\section{Western blot}

Protein isolation from cells, quantification, electrophoresis and electro-blotting of $60 \mu \mathrm{g}$ protein were done as described previously [26]. Unspecific binding of antibodies was blocked using 5\% (w/v) BSA in 2-amino-2-(hydroxymethyl)-propane-1,3-diole-buffered saline (TBS)-T. Polyclonal primary antibodies against murine Adam-10 (R\&D, Minneapolis, USA), Timp-1 (Gillian Murphy, Cambridge, GB), and $\alpha$-Tubulin (Calbiochem Immunochemicals, Darmstadt, Germany) as well as horseradish peroxidaseconjugated secondary antibodies (Amersham Biosciences, Amersham, UK; Invitrogen, Carlsbad, USA) were used for detection with Lumi-light (Roche Diagnostics, Penzberg, Germany).

In vitro assays

$2 \times 10^{6} / \mathrm{ml}$ L-CI.5s knock down or control cells were incubated in serum-free media with or without supplementation of $1000 \mathrm{ng} / \mathrm{ml}$ rec. TIMP-1 for $1 \mathrm{~h}$ and/or $100 \mathrm{ng} / \mathrm{ml} \mathrm{rec} \mathrm{human} \mathrm{Hgf}$ for $10 \mathrm{~min}$. Afterwards cells were spinned on glass slides.

\section{Experimental metastasis assays}

5,000 L-CI.5s cells were injected into the tail vein of pathogen-free syngeneic DBA/2 mice (Charles River, Sulzfeld, Germany). Mice were sacrificed 7 days after tumor cell inoculation and livers were removed. Samples of each liver were snap-frozen in liquid nitrogen for biochemical analysis. Staining of median lobes with 5-bromo-4-chloro-3-indolyl- $\beta$-D-galactopyranoside (X-Gal) (Fermentas, St. Leon-Rot, Germany) was done as described previously [19]. Indigo-blue foci reaching the cutoff-size of
$0.2 \mathrm{~mm}$ were quantified as macrometastases. To determine the total metastatic burden, mRNA expression of the tumour cell tag lacZ was assessed by qRT-PCR as described above. All animal experiments were done in compliance with the guidelines of the Tierschutzgesetz des Landes Bayern.

Immunostaining

Immunostaining of phosphorylated Met was performed as described previously [19, 26]. Simultaneous counterstaining was done with $1 \mu \mathrm{g} / \mathrm{ml} \mathrm{4',6-diamidine-2-phenylindole}$ (DAPI, AppliChem, Darmstadt, Germany) or hematoxylin and sections were mounted with Moviol (Merck). For densitometric analysis signal-intensity of Alexa fluor 488 was measured using ImageJ software.

In situ hybridization

In situ hybridization for murine Timp-1 was done as described previously [18]. Both sense and anti-sense strands were generated (as described before [18]) using bp 5-244 of murine Timp-1. In order to combine X-Galstaining with in situ hybridization, liver pieces were stained with X-Gal prior to paraffin-embedding.

\section{Statistics}

Normal distribution of data was tested using the Kolmogorov-Smirnov test and equal variance was tested using Levene-Median test. Normally distributed data were tested by Student's $t$-test. Otherwise, Mann-Whitney U rank sum test was applied. $P<0.05$ was considered significant.

\section{Results}

Timp-1 expressed by tumor cells within liver metastases

We have previously reported that Timp-1 mRNA expression increases with progression in an experimental liver metastasis assay (L-CI.5s) [13]. In order to elucidate where TIMP-1 expression is localized in L-CI.5s metastasisbearing livers, expression of Timp-1 was localized by in situ hybridization. We found that Timp-1 expression was restricted to metastatic foci (Fig. 1a), indicating a locoregional significance of its expression at this site. In order to elucidate whether tumor cells itself up-regulate Timp-1 expression during metastatic colonization of the liver, we combined in situ hybridization of Timp-1 with X-Galstaining of beta-Galactosidase, which enables us to specifically identify the lacZ-tagged tumor cells in vivo [33]. 
Fig. 1 Timp-1 expressed by tumor cells within liver metastases. a. In situ hybridisation of Timp-1: Timp1 expression is restricted to metastatic foci in tumor cell bearing livers 7 days post i.v. inoculation of 5,000 L-CI.5s tumor cells. Representative picture of metastasis bearing livers. Lower picture

corresponding sense control. Arrow metastatic colonies. Bars $100 \mu \mathrm{m}$. b. Combination of $\mathrm{X}-\mathrm{Gal}$ staining and in situ Hybridisation of Timp-1: tumor cells contribute to formation of local high Timp-1 environments by expression of Timp-1. Indigo blue dot tumor cell. Violet signal Timp-1 expression. Lower picture corresponding sense control. Arrow Timp-1 expressing L-CI.5s tumor cell. Bars $25 \mu \mathrm{m}$
A

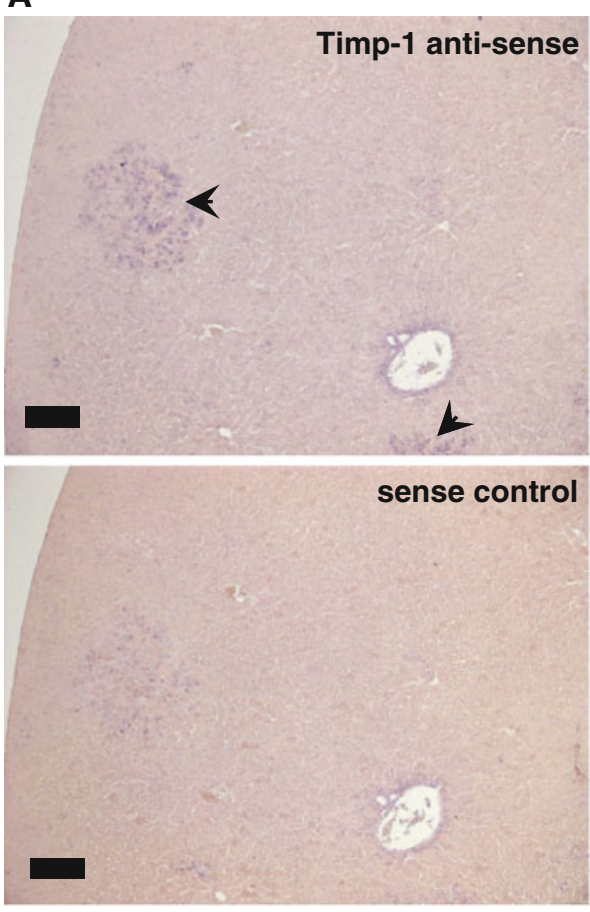

B

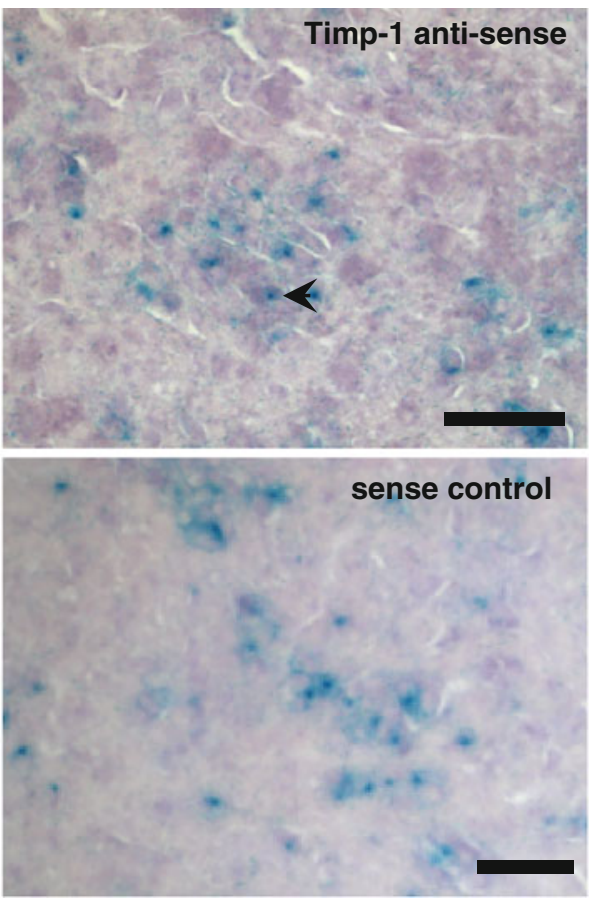

Indeed, detection of co-expression of Timp-1 and betaGalactosidase (Fig. 1b) indicates that tumor cells themselves contribute to the formation of local high Timp-1 environments in metastatic colonies in the liver.

Timp-1 knock down in tumor cells reduced experimental liver metastasis formation

Since high systemic exogenous levels of Timp-1 were shown to promote metastasis $[14,34]$ we hypothesized that tumor cells also take advantage of the endogenous tumor cell-derived Timp-1 expression (Fig. 1). In order to elucidate this question, we created L-CI.5s tumor cells (using lentiviral shRNA technology) which are incapable to induce Timp-1 expression in vivo. It is important to note that Timp-1 mRNA is hardly detectable in vitro (data not shown) but is induced in vivo (Fig. 1b). Therefore, in situ hybridization was employed to detect inhibition of Timp-1 induction. In situ hybridization revealed efficient prevention of Timp-1-induction upon knock down of tumor cellTimp-1 within metastatic foci (Fig. 2a). This result was confirmed by qRT-PCR and Western Blot analysis (Fig. 2b).

In order to analyse the impact of endogenous TIMP-1 expression by tumor cells on metastatic colonization of the liver by L-CI.5s tumor cells, we inoculated L-CI.5s small hairpin (sh)-scrambled and shTimp-1 tumor cells respectively intraveneous (i.v.) in syngeneic mice. Knock down of tumor cell-derived Timp-1 significantly reduced the metastatic potential of the L-CI.5s cells in vivo, as X-Gal staining revealed a decreased number of macro-metastatic colonies in the livers as compared to the control (Fig. 2c, d). The finding that the total tumor burden, shown by RTPCR detection of the lac $Z$ gene tag, was even more strongly reduced (Fig. 2e) indicates that also the scattering of many single tumor cells throughout the liver parenchyma, which is typical for this model $[14,18,19]$, is suppressed.

Timp-1 knock down in tumor cells reduced tumor cell scattering in vivo and Met-signaling activation both in vivo and in vitro

Suppression of scattering of many single tumor cells into the liver parenchyma by knock down of Timp-1 was evident from close-up analysis of X-Gal stained livers (Fig. 3a). In the control group, we could detect single tumor cells scattered throughout the liver parenchyma at day 7 after tumor cell inoculation. In contrast, knock down of tumor cell-derived Timp-1 reduced tumor cell scattering into the liver parenchyma. Previously we have shown that tumor cell scattering of L-CI.5s into the liver parenchyma is mediated by Met-signaling [19]. In the present study we found that knock down of Timp-1 in tumor cells also reduced activation of Met signaling within metastatic foci (Fig. 3b), correlating with reduction of tumor cell scattering (Fig. 3a). In order to analyze this phenomenon in more detail, we incubated the tumor cells in vitro with $\mathrm{SF} / \mathrm{Hgf}$, the ligand of the Met receptor, and analyzed the resulting activation of the Met receptor. L-CI.5s tumor cells react in 
A
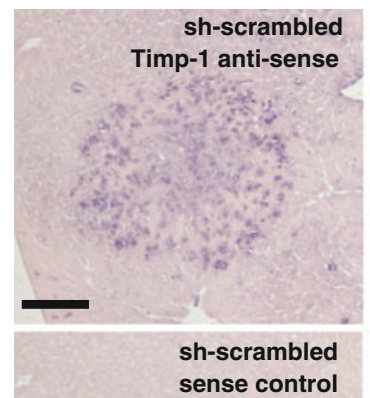
sense control
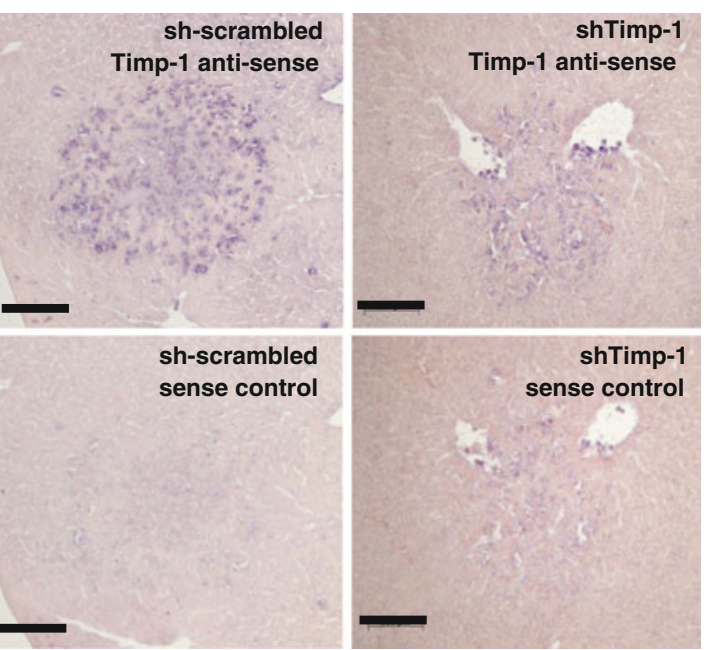

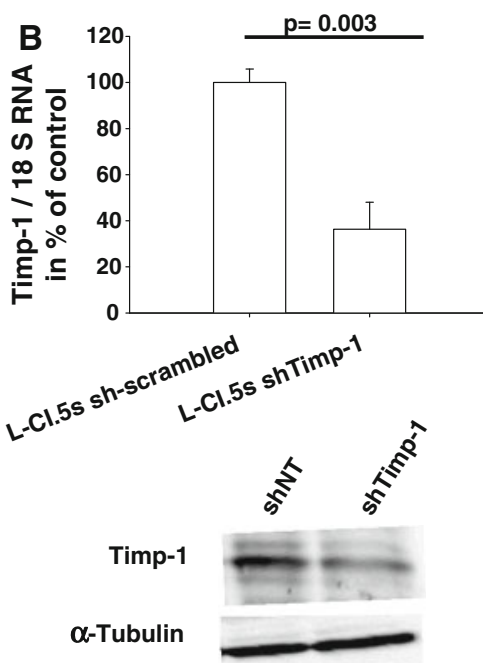

C
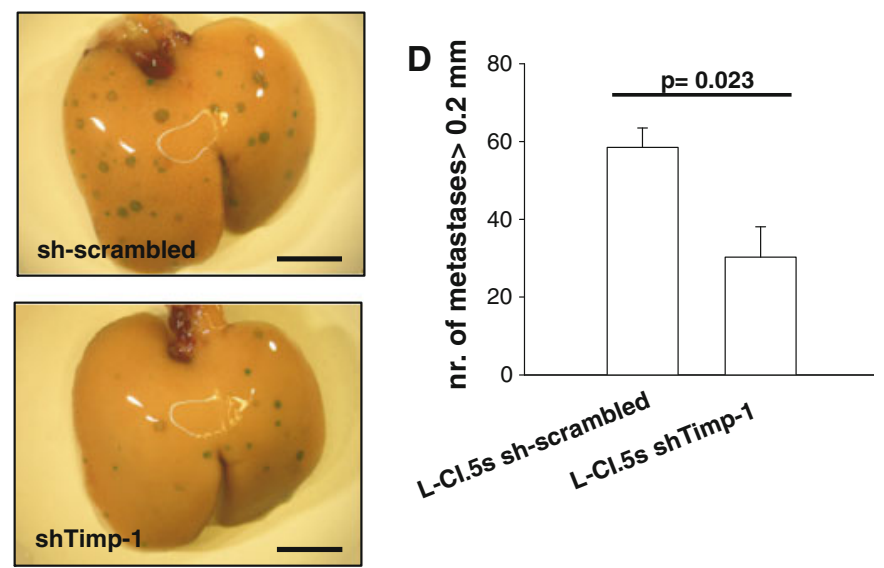

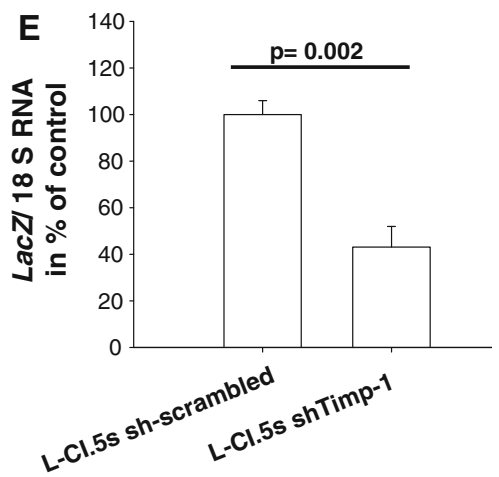

Fig. 2 Timp-1 knock down in tumor cells reduced experimental liver metastasis formation. a. and b. Knock down of Timp-1 in L-CI.5s cells reduced Timp-1 expression in vivo. Timp-1 knock-down cells were generated using lentiviral shRNA technology. a Representative picture of in situ hybridization with corresponding sense controls of Timp-1 7 days after i.v. inoculation of L-CI.5s-sh-scrambled or shTimp-1 respectively. Bars $100 \mu \mathrm{m}$. b qRT-PCR and representative Western Blot of Timp-1 7 days after i.v. inoculation of L-CI.5s-shscrambled or shTimp-1 respectively. L-CI.5s sh-scrambled: $100.0 \pm 5.8, n=4$; L-CI.5s shTimp-1: $36.3 \pm 11.7, n=4$. Data was normally distributed. c Timp-1 knock down reduced

vitro upon Hgf stimulation with increased phosphorylation of the Met receptor (Fig. 3c). This increase of liganddependent Met-phosphorylation was inhibited by knock down of tumor cell-derived Timp-1 (Fig. 3c).

Inhibition with Timp-1 or knock down of tumor cellderived Adam-10 increases Met-phosphorylation in vitro

As we have shown that tumor cells are suppressed to respond to SF/Hgf upon knock down of Timp-1, endogenous Timp-1 must be involved in the regulation of Met experimental metastasis-formation. X-Gal-stained livers 7 days after i.v. inoculation of L-CI.5s-sh-scrambled or shTimp-1 respectively. d Quantification of liver metastasis $>0.2 \mathrm{~mm}$ livers 7 days after i.v. inoculation of L-CI.5s-sh-scrambled or shTimp-1 respectively. L-CI.5s sh-scrambled: $58.5 \pm 5.0, \quad n=4 ; \quad$ L-CI.5s shTimp-1: $30.3 \pm 7.8, n=4$. Data was normally distributed. e Total tumor burden in the liver assessed by qRT-PCR of the tumor cell marker lacZ normalized to $18 \mathrm{~S}$ RNA expression 7 days after i.v. inoculation of L-CI.5s-sh-scrambled or shTimp-1 respectively. L-CI.5s shscrambled: $100.0 \pm 5.9, n=4 ; \quad$ L-CI.5s shTimp-1: $43.0 \pm 8.9$, $n=4$. Data was normally distributed

signaling at the tumor cell surface. As we have previously shown that the Timp-1-sensitive protease Adam-10 is a Sheddase of the Met-receptor [26], we next wanted to know whether Timp-1 acts via this mechanism. To this end, we investigated SF/Hgf-induced Met phosphorylation when Adam-10 was inhibited by rec Timp-1 (Fig. 4a) or when Adam-10 was specifically knocked down by shRNA (Fig. 4c). L-CI.5s tumor cells react upon Hgf stimulation with increased phosphorylation of the Met receptor (Fig. 4a, c). Timp-1 incubation increased Met phosphorylation in presence as well as in absence of SF/Hgf (Fig. 4a). Also, the specific knock down of Adam-10 (knock down 
A

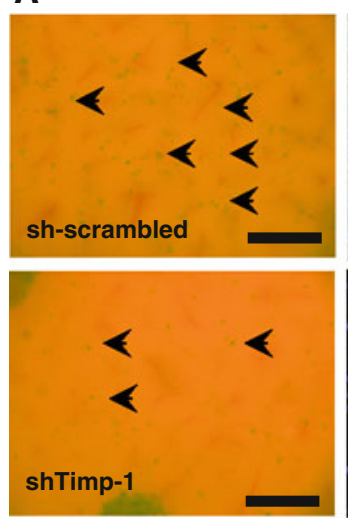

C
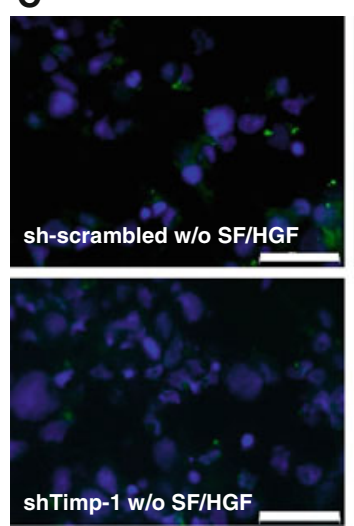

B

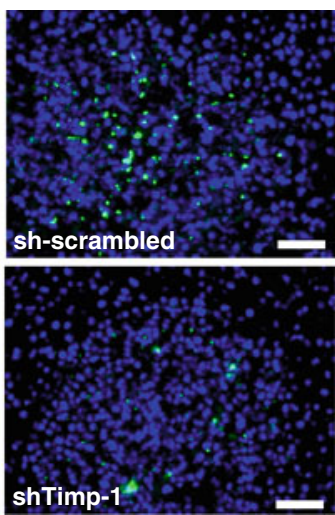

shTimp-1 to

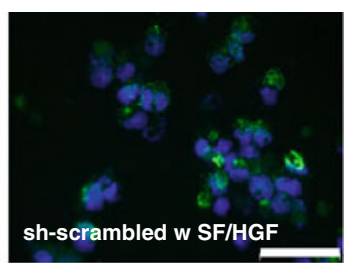

Fig. 3 Timp-1 knock down in tumor cells reduced tumor cell scattering in vivo and Met-signaling activation both in vivo and in vitro. a. Timp-1 knock down in tumor cells reduced single tumor cell scattering. Representative close-up pictures of X-Gal-stained livers 7 days after i.v. inoculation of L-CI.5s-sh-scrambled or shTimp-1 respectively. Indigo blue dots tumor cells. Bars $0.2 \mathrm{~mm}$. b Immunohistochemical staining for phosphorylated Met inside liver metastases 7 days after i.v. inoculation of L-CI.5s-sh-scrambled or shTimp-1 respectively. Timp-1 knock down reduced phosphorylation of Met receptors (green signal) inside metastatic foci. Counter stain: DAPI

efficiency on mRNA level: 89\%, Western Blot analysis of knock down efficiency (Fig. 4b)) led to an increased response to SF/Hgf (Fig. 4c). Also here, at least a trend of increase in ligand-independent Met phosphorylation was observed (Fig. 4c). This was in accordance with our previous findings $[14,26]$ that the Timp-1-sensitive Sheddase Adam-10 is an important regulator of Met-signalingregulation.

Knock down of tumor cell-derived Adam-10 increases Met-phosphorylation and tumor cell scattering in vivo

In order to investigate the role of Adam-10 in this context further, we next tested the effect of Adam-10 knock down on phosphorylation of the Met receptor in vivo within metastatic foci. As expected, knock down of Adam-10

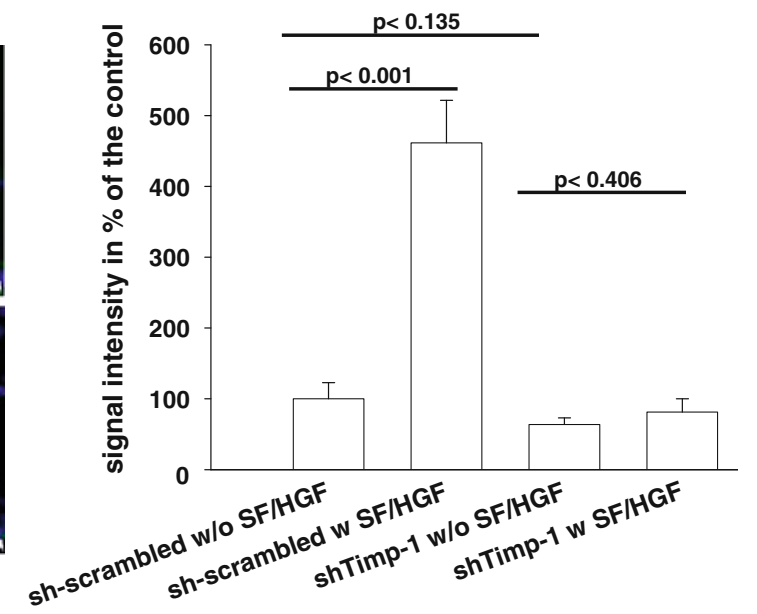

(blue signal). Bars $25 \mu \mathrm{m}$. c Knock down of Timp-1 reduced susceptibility to the Met ligand Hgf. Representative pictures of immunocytochemical staining for phosphorylated Met (green signal) in L-CI.5s-sh-scrambled and L-CI.5s-shTimp-1 cells. Cells were stimulated with $100 \mathrm{ng} / \mathrm{ml}$ Hgf. Counter stain: DAPI (blue signal). Bars $50 \mu \mathrm{m}$. Right panel corresponding densitometric analysis of Met phosphorylation. $n=20$ cells each; L-CI.5s-sh-scrambled w/o Hgf: $100.0 \pm 22.9 \%$; L-CI.5s-sh-scrambled w Hgf: $461.4 \pm 60.3 \%$; L-CI.5s-sh-Timp-1 w/o Hgf: $63.5 \pm 9.6 \%$; L-CI.5s-sh-Timp-1 w Hgf: $81.3 \pm 18.8 \%$. Data was normally distributed

increased Met phosphorylation as compared to the control group (Fig. 5a). This increase was coincident with elevation of scattered liver metastasis of L-CI.5s cells with Adam-10 knock down (Fig. 5b). While the number of macro-metastatic colonies was significantly decreased upon knock down of Timp-1 in the tumor cells (Fig. 5c), the total metastatic burden was unaltered (Fig. 5d), reflecting a change of pattern of metastasis towards the scattered phenotype.

\section{Discussion}

In the present study we revealed that tumor cell-derived Timp-1 regulates Met-dependent tumor cell scattering in 

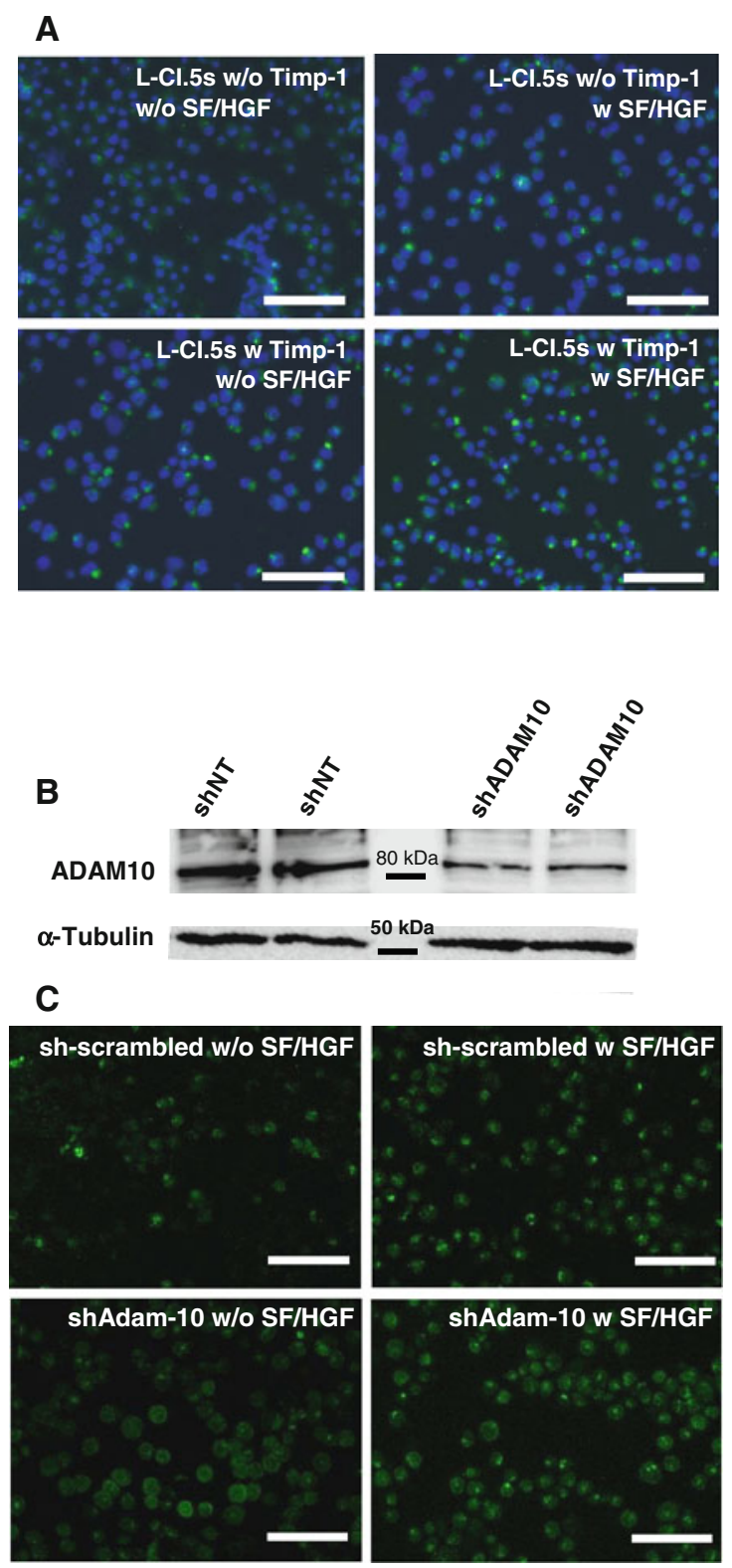

Fig. 4 Inhibition of tumor cell-derived Adam-10 by Timp-1 or Adam10 knock down increases Met-phosphorylation in vitro. a Inhibition of Adam-10 by exogenous Timp-1 increased ligand-independent phosphorylation and susceptibility of L-CI.5s cells to Hgf in vitro. Immunocytochemistry for phosphorylated Met (green signal) in L-CI.5s tumor cells in vitro. Cells were either unstimulated or incubated with $1000 \mathrm{ng} /$ $\mathrm{ml}$ rec. Timp-1. Phosphorylation of the Met receptor was stimulated with $100 \mathrm{ng} / \mathrm{ml}$ Hgf. Counter stain: DAPI (blue signal). Bars $50 \mu \mathrm{m}$. Right panel: corresponding densitometric analysis of Met phosphorylation. $n=20$ cells each; L-CI.5s w/o Timp-1 w/o Hgf: $100.0 \pm 8.0 \%$; L-CI.5s w/o Timp-1 w Hgf: $153.7 \pm 13.0 \%$; L-CI.5s w Timp-1 w/o Hgf : $207.5 \pm 20.8 \%$; L-CI.5s w Timp-1 w Hgf: $277.4 \pm 26.9 \%$. Data was not

the liver. In addition we have evidence that Adam-10 is involved in this process. This reasoning is supported by the fact that Timp-1 is an inhibitor of Adam-10 [25], which was recently shown to be a sheddase of the Met tyrosine kinase receptor [26, 27].
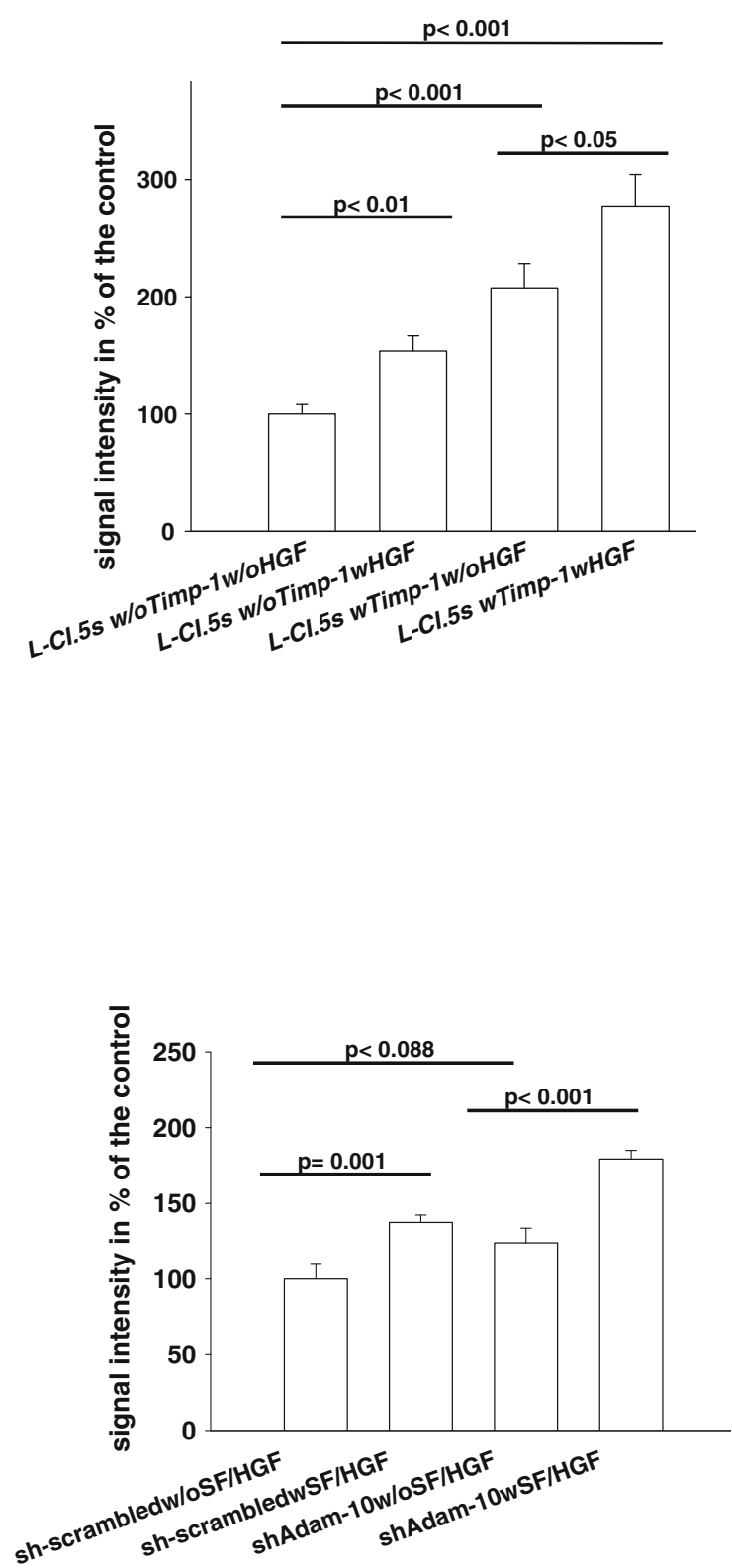

normally distributed. b and c Loss of Adam-10 increased ligandindependent phosphorylation and susceptibility of L-CI.5s cells to Hgf in vitro. b Representative Western Blot analysis of ADAM-10 knock down in L-CI.5s tumor cells. c Immunocytochemistry for phosphorylated Met (green signal) in L-CI.5s-sh-scrambled and shAdam-10 tumor cells in vitro. Control cells were unstimulated, whereas phosphorylation in the second group was stimulated with $100 \mathrm{ng} / \mathrm{ml} \mathrm{Hgf.} \mathrm{Bars} 50 \mu \mathrm{m}$. Right panel: corresponding densitometric analysis of Met phosphorylation. $n=20$ cells each; L-CI.5s-sh-scrambled w/o Hgf: $100.0 \pm 9.7 \%$; L-CI.5s-sh-scrambled w Hgf: $137.5 \pm 4.8 \%$; L-CI.5s-shAdam-10 w/o Hgf: $124.0 \pm 9.6 \%$; L-CI.5s-shAdam-10 w Hgf: $179.3 \pm 5.7 \%$. Data was normally distributed

This decisively broadens the knowledge on molecular mechanisms explaining the counter-intuitive finding that Timp-1 levels correlate with bad prognosis [1-4]. What first seemed to be a paradox, as protease inhibitors were long thought to only play a role in inhibiting the pro-metastatic 

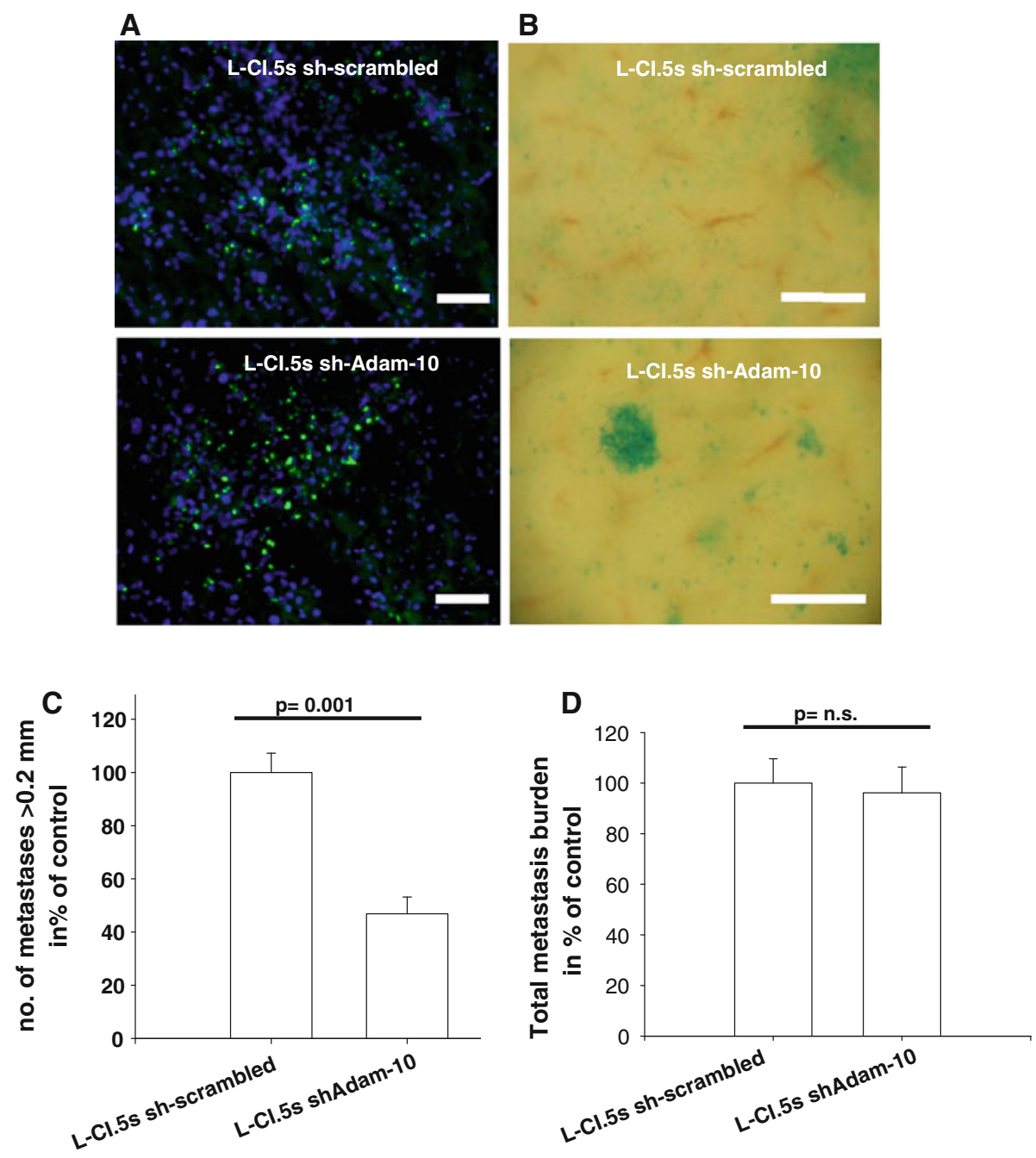

Fig. 5 Knock down of tumor cell-derived Adam-10 increases Met phosphorylation and tumor cell scattering in vivo. a Knock down of Adam-10 increased Met phosphorylation inside liver metastases. Immunohistochemical staining for phosphorylated Met (green signal) inside liver metastases 7 days after i.v. inoculation of L-CI.5s-shscrambled or shAdam-10 respectively. Counter stain: DAPI (blue signal). Bars $25 \mu \mathrm{m}$. b Adam-10 knock down in tumor cells reduced single tumor cell scattering. Representative close-up pictures of $\mathrm{X}$-Gal-stained livers 7 days after i.v. inoculation of L-CI.5s-sh-

function of ECM-degrading activity of tumor-associated proteases [35, 36], including MMPs [36], now, with the advent of the concept of the protease web, has turned out to be quite logical. The protease web describes the complexity of the effects of proteolytic activity, which decisively regulate cell signaling rather than just degrading components of the ECM $[16,17]$. This implies that the biological function of many proteases had to be revised $[10,11]$. Similarly, the role of protease inhibitors, which regulate all these processes, has been under revision in the past years [14, 37]. Early studies reported that metastatic colonization is increased in mice when host Timp-1 was decreased upon expression of anti-sense RNA in transgenic

scrambled or shAdam-10 respectively. Indigo blue dots tumor cells. Bars $0.2 \mathrm{~mm}$. c Quantification of liver metastasis $>0.2 \mathrm{~mm}$ livers 7 days after i.v. inoculation of L-CI.5s-sh-scrambled or shAdam-10 respectively. L-CI.5s sh-scrambled: $100.0 \pm 7.2 \%, n=4$; L-CI.5s shAdam-10: $46.8 \pm 6.3 \%, n=4$. Data was normally distributed. d Total metastasis burden in the liver in $\%$ of the control 7 days after i.v. inoculation of L-CI.5s-sh-scrambled or shAdam-10 respectively. L-CI.5s sh-scrambled: $100.0 \pm 9.7 \%, n=4$; L-CI.5s shAdam-10: $96.1 \pm 10.2 \%, n=4$. Data was normally distributed

mice [38]. Furthermore very high systemic levels of Timp1 , achieved by adenoviral gene transfer protect the liver from metastatic colonization by a T-cell lymphoma [39]. Surprisingly, slightly lower levels of Timp-1, also achieved upon adenoviral gene transfer, increase the susceptibility of the liver to tumor cells [14], indicating the complexity of the checks and balances of the proteolytic network [16] on the tissue level, which may be concentration dependent as the anti-proteolytic activity and anti-extravasatory activity may become dominant over secondary signaling effects [14]. The central mechanism of this Timp-1-associated promotion of metastasis is the induction of Metsignaling in the liver tissue [14] leading to an alteration of 
the gene expression signature which included up-regulation of a variety of pro-metastatic genes [14]. Activation of Met-signaling is associated with elevated levels of Metreceptor on the surface of the liver cells and we had suggested that Timp-1 leads to this accumulation by inhibiting Adam-10 [14]. Indeed, we and others could recently show that Adam-10 is a sheddase of the Met-receptor [26, 27]. All these studies, showing either metastasis promotion or suppression by Timp-1, indicate that Timp-1 can play different roles during metastasis depending on the level of expression and the site of action.

The present study was intrigued by our initial finding that Timp-1 expression was observed in the metastatic colonies of the L-CI.5s tumor model. This seemed paradox as we have previously found that in this model MMP-2 is important for colony outgrowth and MMP-9 is important for maintaining the metastatic potential [18]. Both proteases are effectively inhibited by Timp-1 [40]. Therefore, Timp-1 must be involved in a molecular mechanism which is at least evenly important. Indeed, we could here show that Timp-1, expressed by tumor cells, was crucial for maintaining Met-signaling activation in tumor cells and promoting metastatic scattering. Previous results have shown that Met is essential for single tumor cell scattering of L-CI.5s cells into the liver parenchyma [19]. Interestingly, knock down of Timp-1 in tumor cells led to a similar phenotype, suggesting an important role of Met also in this context. This is even more obvious as we observed a strong reduction of Met signaling activation within metastatic foci caused by knock down of Timp-1 in tumor cells. It is already well established that Met signaling is mainly regulated by presence of the receptor at the cell surface [22, $26]$ and that ADAMs play a crucial role in this process [14, 26, 41]. So far, Adam-10 and also ADAM-17 were found to be able to shed the Met receptor thereby regulating Metsignaling activity. As only Adam-10 but not Adam-17 is inhibited by Timp-1 [25, 42] the involvement of Adam-17 in the described setting could be excluded. The importance of Adam-10 in regulating the Met signaling pathway was stressed by our finding that in vivo knock down of Adam10 in L-CI.5s tumor cells led to induction of Met phosphorylation. The fact that knock down of Adam-10 led to reduction of macro-metastases but did not alter total tumor burden is a clear indication of increased metastatic spread of single cells throughout the liver parenchyma. This scattered infiltration of the liver is also observed in situations where Adam-10 is inhibited by high systemic levels of Timp-1 [14]. In both cases, increased Met signaling correlated with increased metastatic infiltration of the whole liver parenchyma, a situation which would lead to unresectable hepatic metastases and bad prognosis for cancer patients [28]. Taken together, these results show for the first time in vivo that Adam-10 can also act as an anti- metastatic protein by controlling the metastasis-promoting Met signaling pathway, while it is also discussed that Adam-10 can act as a pro-metastatic protein and therapeutic strategies based on its inhibition have been envisaged [43].

From clinical samples it is not really clear which entity of the tumor expresses Timp-1 [44]. In principal both stromal cells and tumor cells were found to express Timp-1 in clinical samples. However, the main source for Timp-1 seems to be the stromal compartment [44]. The present study shows that in principal also tumor cells can express Timp-1 thereby increasing their metastatic potential. However, this does not exclude that also expression of Timp-1 by stromal cells can act in trans and activate the Met pathway in tumor cells thereby increasing their metastatic potential.

In conclusion, our study strongly points at the necessity to carefully unravel the complexity of the biology of the protease web in order to avoid surprises when pushing molecules of the network as targets into the clinic.

Acknowledgments We thank Katja Honert and Stefan Grötzinger (all from Institut für Experimentelle Onkologie und Therapieforschung des Klinikums rechts der Isar, Technische Universität München, Munich, Germany) for their expert technical assistance and Gillian Murphy for providing the anti-Timp-1 antibody. For financial support, the authors thank the European Union Research Framework Programme 7, project HEALTH-2007-201279/Microenvimet (to Achim Krüger, Carla Boccaccio and Paolo Comoglio).

\section{References}

1. Terpos E, Dimopoulos MA, Shrivastava V et al (2010) High levels of serum TIMP-1 correlate with advanced disease and predict for poor survival in patients with multiple myeloma treated with novel agents. Leuk Res 34(3):399-402

2. Aaberg-Jessen C, Christensen K, Offenberg H et al (2009) Low expression of tissue inhibitor of metalloproteinases-1 (TIMP-1) in glioblastoma predicts longer patient survival. J Neurooncol 95(1): 117-128

3. Scrideli CA, Cortez MA, Yunes JA et al (2010) mRNA expression of matrix metalloproteinases (MMPs) 2 and 9 and tissue inhibitor of matrix metalloproteinases (TIMPs) 1 and 2 in childhood acute lymphoblastic leukemia: potential role of TIMP1 as an adverse prognostic factor. Leuk Res 34(1):32-37

4. Rauvala M, Puistola U, Turpeenniemi-Hujanen T (2005) Gelatinases and their tissue inhibitors in ovarian tumors; TIMP- 1 is a predictive as well as a prognostic factor. Gynecol Oncol 99(3):656-663

5. Chirco R, Liu XW, Jung KK et al (2006) Novel functions of TIMPs in cell signaling. Cancer Metastasis Rev 25(1):99-113

6. Mook OR, Frederiks WM, Van Noorden CJ (2004) The role of gelatinases in colorectal cancer progression and metastasis. Biochim Biophys Acta 1705(2):69-89

7. Egeblad M, Werb Z (2002) New functions for the matrix metalloproteinases in cancer progression. Nat Rev Cancer 2(3): $161-174$

8. Coussens LM, Fingleton B, Matrisian LM (2002) Matrix metalloproteinase inhibitors and cancer: trials and tribulations. Science 295(5564):2387-2392 
9. Deryugina EI, Quigley JP (2006) Matrix metalloproteinases and tumor metastasis. Cancer Metastasis Rev 25(1):9-34

10. Gutierrez-Fernandez A, Fueyo A, Folgueras AR et al (2008) Matrix metalloproteinase-8 functions as a metastasis suppressor through modulation of tumor cell adhesion and invasion. Cancer Res 68(8):2755-2763

11. Garg P, Sarma D, Jeppsson S et al (2010) Matrix metalloproteinase- 9 functions as a tumor suppressor in colitis-associated cancer. Cancer Res 70(2):792-801

12. Krüger A et al (2001) Hydroxamate-type matrix metalloproteinase inhibitor batimastat promotes liver metastasis. Cancer Res 61(4):1272-1275

13. Schelter F, Halbgewachs B, Baumler P et al (2011) Tissue inhibitor of metalloproteinases-1-induced scattered liver metastasis is mediated by hypoxia-inducible factor-1alpha. Clin Exp Metastasis 28(2):91-99

14. Kopitz C, Gerg M, Bandapalli OR et al (2007) Tissue inhibitor of metalloproteinases-1 promotes liver metastasis by induction of hepatocyte growth factor signaling. Cancer Res 67(18):8615-8623

15. Krüger A (2009) Functional genetic mouse models: promising tools for investigation of the proteolytic internet. Biol Chem 390(2):91-97

16. Overall CM, Kleifeld O (2006) Tumour microenvironmentopinion: validating matrix metalloproteinases as drug targets and anti-targets for cancer therapy. Nat Rev Cancer 6(3):227-239

17. Krüger A, Kates RE, Edwards DR (2010) Avoiding spam in the proteolytic internet: future strategies for anti-metastatic MMP inhibition. Biochim Biophys Acta 1803(1):95-102

18. Gerg M, Kopitz C, Schaten S et al (2008) Distinct functionality of tumor cell-derived gelatinases during formation of liver metastases. Mol Cancer Res 6(3):341-351

19. Schelter F, Gerg M, Halbgewachs B et al (2010) Identification of a survival-independent metastasis-enhancing role of hypoxiainducible factor-1alpha with a hypoxia-tolerant tumor cell line. J Biol Chem 285(34):26182-26189

20. Naldini L, Weidner KM, Vigna E et al (1991) Scatter factor and hepatocyte growth factor are indistinguishable ligands for the MET receptor. EMBO J 10(10):2867-2878

21. Boccaccio C, Comoglio PM (2006) Invasive growth: a METdriven genetic programme for cancer and stem cells. Nat Rev Cancer 6(8):637-645

22. Trusolino L, Bertotti A, Comoglio PM (2010) MET signalling: principles and functions in development, organ regeneration and cancer. Nat Rev Mol Cell Biol 11(12):834-848

23. Comoglio PM, Giordano S, Trusolino L (2008) Drug development of MET inhibitors: targeting oncogene addiction and expedience. Nat Rev Drug Discov 7(6):504-516

24. Murphy G (2008) The ADAMs: signalling scissors in the tumour microenvironment. Nat Rev Cancer 8(12):929-941

25. Amour A, Knight CG, Webster A et al (2000) The in vitro activity of ADAM-10 is inhibited by TIMP-1 and TIMP-3. FEBS Lett 473(3):275-279

26. Schelter F, Kobuch J, Moss ML et al (2010) A disintegrin and metalloproteinase-10 (ADAM-10) mediates DN30 antibodyinduced shedding of the met surface receptor. J Biol Chem 285(34):26335-26340
27. Schirrmeister W, Gnad T, Wex T et al (2009) Ectodomain shedding of E-cadherin and c-Met is induced by Helicobacter pylori infection. Exp Cell Res 315(20):3500-3508

28. Koshariya M, Jagad RB, Kawamoto J et al (2007) An update and our experience with metastatic liver disease. Hepatogastroenterology 54(80):2232-2239

29. Sporn MB (1996) The war on cancer. Lancet 347(9012): $1377-1381$

30. Hanahan D, Weinberg RA (2011) Hallmarks of cancer: the next generation. Cell 144(5):646-674

31. Krüger A, Schirrmacher V, von Hoegen P (1994) Scattered micrometastases visualized at the single-cell level: detection and reisolation of lacZ-labeled metastasized lymphoma cells. Int $\mathrm{J}$ Cancer 58(2):275-284

32. Soneoka Y, Cannon PM, Ramsdale EE et al (1995) A transient three-plasmid expression system for the production of high titer retroviral vectors. Nucleic Acids Res 23(4):628-633

33. Krüger A, Schirrmacher V, Khokha R (1998) The bacterial lacZ gene: an important tool for metastasis research and evaluation of new cancer therapies. Cancer Metastasis Rev 17(3):285-294

34. Schrötzlmair F, Kopitz C, Halbgewachs B et al (2010) Tissue inhibitor of metalloproteinases-1-induced scattered liver metastasis is mediated by host-derived urokinase-type plasminogen activator. J Cell Mol Med 14(12):2760-2770

35. Brand $\mathrm{K}$ et al (2000) Treatment of colorectal liver metastases by adenoviral transfer of tissue inhibitor of metalloproteinases- 2 into the liver tissue. Cancer Res 60(20):5723-5730

36. Baker AH, Edwards DR, Murphy G (2002) Metalloproteinase inhibitors: biological actions and therapeutic opportunities. J Cell Sci 115(Pt 19):3719-3727

37. Bajou $\mathrm{K}$ et al (1998) Absence of host plasminogen activator inhibitor 1 prevents cancer invasion and vascularization. Nat Med 4(8):923-928

38. Krüger A, Fata JE, Khokha R (1997) Altered tumor growth and metastasis of a T-cell lymphoma in Timp-1 transgenic mice. Blood 90(5):1993-2000

39. Elezkurtaj S, Kopitz C, Baker AH et al (2004) Adenovirusmediated overexpression of tissue inhibitor of metalloproteinases-1 in the liver: efficient protection against T-cell lymphoma and colon carcinoma metastasis. J Gene Med 6(11):1228-1237

40. Brew K, Nagase H (2010) The tissue inhibitors of metalloproteinases (TIMPs): an ancient family with structural and functional diversity. Biochim Biophys Acta 1803(1):55-71

41. Foveau B et al (2009) Down-regulation of the met receptor tyrosine kinase by presenilin-dependent regulated intramembrane proteolysis. Mol Biol Cell 20(9):2495-2507

42. Amour A et al (1998) TNF-alpha converting enzyme (TACE) is inhibited by TIMP-3. FEBS Lett 435(1):39-44

43. Moss ML, Stoeck A, Yan W et al (2008) ADAM10 as a target for anti-cancer therapy. Curr Pharm Biotechnol 9(1):2-8

44. Würtz SO, Schrohl AS, Sorensen NM et al (2005) Tissue inhibitor of metalloproteinases-1 in breast cancer. Endocr Relat Cancer 12(2):215-227 